\title{
Evaluating the design of inclusive interfaces by simulation
}

\author{
Pradipta Biswas \\ Computer Laboratory \\ $15 \mathrm{JJ}$ Thomson Avenue \\ Cambridge CB3 0FD \\ University of Cambridge, UK \\ E-mail:pb400@cam.ac.uk
}

\author{
Peter Robinson \\ Computer Laboratory \\ 15 JJ Thomson Avenue \\ Cambridge CB3 0FD \\ University of Cambridge, UK \\ E-mail:pr10@cam.ac.uk
}

\begin{abstract}
We have developed a simulator to help with the design and evaluation of assistive interfaces. The simulator can predict possible interaction patterns when undertaking a task using a variety of input devices, and estimate the time to complete the task in the presence of different disabilities. We have evaluated the simulator by considering a representative application being used by able-bodied, visually impaired and mobility impaired people. The simulator predicted task completion times for all three groups with statistically significant accuracy. The simulator also predicted the effects of different interface designs on task completion time accurately.
\end{abstract}

\section{Categories and Subject Descriptors}

D.2.2 [Software Engineering]: Design Tools and Techniques - user interfaces; K.4.2 [Computers and Society]: Social Issues - assistive technologies for persons with disabilities

\section{General Terms}

Algorithms, Experimentation, Human Factors, Measurement

\section{Keywords}

Human Computer Interaction, Assistive Technology, User Model, Usability Evaluation, Simulator.

\section{INTRODUCTION}

The World Health Organisation (WHO) states that the number of people aged 60 and over will be 1.2 billion by 2025 and 2 billion by 2050 [48]. The very old (age $80+$ ) is the fastest growing population group in the developed world. Many of these elderly people have disabilities

\footnotetext{
Permission to make digital or hard copies of part or all of this work for personal or classroom use is granted without fee provided that copies are not made or distributed for profit or commercial advantage and that copies bear this notice and the full citation on the first page. Copyrights for components of this work owned by others than ACM must be honored. Abstracting with credit is permitted. To copy otherwise, to republish, to post on servers or to redistribute to lists, requires prior specific permission and/or a fee.

IUII 2010

Copyright 2008 ACM 978-1-59593-987-6/ 08/ $0001 \$ 5.00$
}

which make it difficult for them to use computers. The definition of the term 'Disability' differs across countries and cultures, but the World Bank [49] estimates a rate of $10-12 \%$ of population worldwide having a condition that inhibits their use of standard computer systems. There are ethical and legislative reasons for designing products and services for this vast population. In particular, computers offer valuable assistance to people with physical disabilities and help to improve their quality of life. However the diverse range of abilities complicates the designing of human-computer interfaces for these users. Existing inclusive or assistive software systems often address a specific class of user and thus many elderly people and users with disabilities find existing technologies hard to use.

We have taken a novel approach to designing and evaluating inclusive systems by modelling performance of users with a wide range of abilities. In this paper, we present a simulator that can predict possible interaction patterns when undertaking a task using a variety of input devices, and estimate the time to complete the task in the presence of different disabilities and for different levels of skill. We briefly describe the simulator and demonstrate its use in evaluating interfaces for an application used by ablebodied, visually impaired and mobility impaired people.

In the next section, we present a brief background of our research. We describe the simulator in Section 3, followed by our study in Section 4. Finally, we summarize the study in Section 5 and also discuss the implications and limitations.

\section{BACKGROUND}

\section{Problem in existing systems}

Researchers in assistive technology have concentrated on designing novel interfaces for many different applications including

- Web Browsers [20, 44].

- Augmentative and alternative communication aids $[2,29,35,45]$.

- New interaction techniques

- Scanning interfaces $[30,33,46]$, 
○ Gravity wells [19].

- Novel hardware interfaces [1]

○ Eye-gaze trackers [28],

○ Brain-computer interfaces $[17,25]$.

However, there is no systematic modelling tool for assistive interfaces. Moreover very few human-computer interaction (HCI) models have considered users with disabilities. Two main types of user model are in widespread use:

- The GOMS family of models, which were developed only for HCI.

- Models involving cognitive architectures, which take a detailed view of human cognition.

The GOMS (Goal, Operator, Model, Selection) family of HCI models (e.g. KLM, CMN-GOMS, CPM-GOMS) is mainly suitable for modelling the optimal (skilled) behaviour of users $[11,21]$. On the other hand, models developed using cognitive architectures consider the uncertainty of human behaviour in detail but have not been widely adopted for simulating HCI as their use demands a detailed knowledge of psychology. A brief review on different types of user models can be found from our earlier publication [7].

A modelling tool for people with disabilities is particularly important, as user trials are often difficult and time consuming. Lack of knowledge about the problems of disabled and elderly users has often led designers to develop non-inclusive systems. There are guidelines for designing accessible systems (particularly accessible websites), but designers often do not conform to the guidelines while developing new systems. Additionally, the guidelines are not adequate to relate the inclusive features of a system with the particular need of users.

Evaluation of assistive interfaces can be even more difficult than their design. Assistive interfaces are generally evaluated by analysing log files after a user trial $[18,26$, 34]. As an example of a different approach, Rizzo and colleagues [39] evaluated the AVANTI project [44], by a technique combining cognitive walkthrough and Normans' seven-stage model [43]. However it is often difficult to find participants with specific disabilities. Petrie and colleagues [36] take the approach of remote evaluation but can not avoid the need to find disabled participants.

\section{Proposed solution}

We take a novel approach to the design and evaluation of assistive interfaces by simulating interaction patterns of users with and without disabilities. Figure 1 shows the use of our models. Our aim is to help evaluate existing systems and different design alternatives with respect to many types of disability. The evaluation process will be used to select a particular interface, which can then be validated by a formal user trial. The user trials also provide feedback to the models to increase their accuracy. As each alternative design does not need to be evaluated by a user trial, it will reduce the development time significantly.

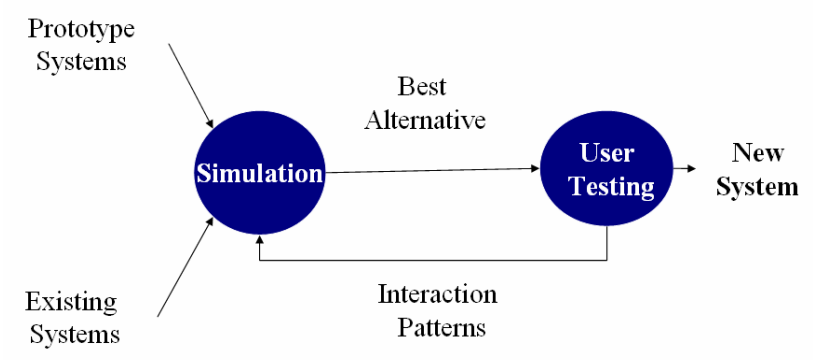

Figure 1. Use of the simulator

Within this framework, our objectives were to develop a model that can:

- Simulate HCI of both able-bodied and disabled users.

- Work for users with different levels of skill.

- Be easy to use and comprehend for an interface designer.

\section{Scope of the paper}

In the next section we briefly present the architecture of our simulator. Previous papers have discussed the design, calibration and validation of the individual components of the simulator [5, 7, 8, and 9]. This study uses the simulator as a whole to model an icon searching task. We have investigated the effects of icon layout and font size of captions on people with visual and motor impairment. We have analyzed the performance of the simulator in predicting the effects of different interface designs on interaction. The study validates the models for an externally valid task and also demonstrates the application of the simulator to compare different interface layouts for people with a wide range of abilities.

\section{THE SIMULATOR}

\section{Architecture of the simulator}

The simulator takes a task definition and locations of different objects in an interface as input. It then predicts possible eye movements and cursor paths on the screen and uses these to predict task completion times. The models are parameterized to represent different physical abilities, levels of skill and input devices.

The architecture of the simulator is shown in Figure 2. It consists of the following three components:

The Application model represents the task currently undertaken by the user by breaking it up into a set of simple 
atomic tasks.

The Interface Model handles different types of input and output device and sets parameters for an interface.

The User Model simulates the interaction patterns of an individual undertaking a task analysed by the task model using the configuration set by the interface model. It uses the sequence of phases defined by Model Human Processor. The perception model simulates the visual perception of interface objects. The cognitive model takes the output of the perception model and determines an action to accomplish the current task. The motor-behaviour model predicts the completion time and possible interaction patterns for performing that action. Our previous papers describe a general case study [5] and an application of the model in evaluating different single-switch scanning techniques [6].

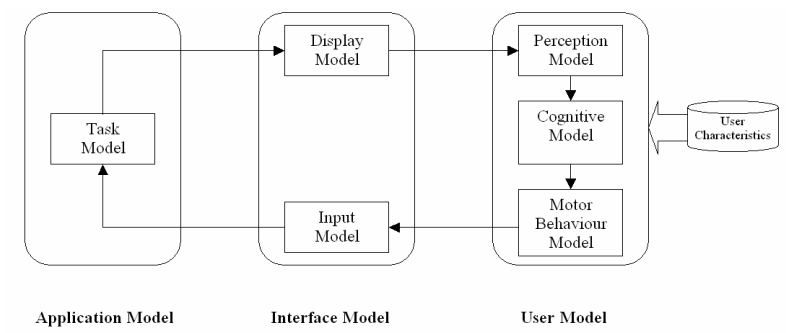

Figure 2. Architecture of the Simulator

The perception model is designed according to the theories of visual attention. Our cognitive model is more detailed than the GOMS model but not as complex as existing cognitive architectures. The motor-behaviour model is based on a statistical analysis of screen navigation paths of disabled user. We present brief descriptions of these models in the following sections.

\section{The perception model}

Our perception model takes a list of mouse events, a sequence of bitmap images of an interface and locations of different objects in the interface as input, and produces a sequence of eye-movements as output [9].

Our model follows the 'spotlight' metaphor of visual perception. Our model works in three steps.

1. Scanning the screen and decomposing it into primitive features.

2. Finding the probable points of attention fixation by evaluating the similarity of different regions of the screen to the one containing the target.

3. Deducing a possible trajectory of eye movement.

The model scans the whole screen by dividing it into several focus rectangles, one of which should contain the actual target. The probable points of attention fixation are calculated by evaluating the similarity of other focus rectangles to the one containing the target. We know which focus rectangle contains the target from the list of mouse events that was input to the system. Similarity is calculated by a neural network, which takes the colour histogram [32] and shape context coefficients [3, 4] of the target and other focus rectangles as input and gives the probable points of fixation as output. The neural network was trained by tracking eye gazes of users with and without visual impairment.

After deducing the points of fixations, the model shifts attention among the points of fixation by combining different eye movement strategies (such as Nearest [16], Cluster $[13,14]$ etc.). We found that the following eye movement strategy worked well for most participants.

- Cluster the probable points of fixations according to their positions

- Launch the first saccade at the centre of the biggest cluster

- Shift attention to the nearest possible points of fixation thereafter.

Our model can also simulate the effect of visual impairment on interaction by modifying the input bitmap images according to the nature of the impairment (such as blurring for visual acuity loss, changing colours for colour blindness). We have also simulated early and late stages of Maccular Degeneration, Diabetic Retinopathy, Glaucoma and different types of colour blindness [9].

We validated the model by a visual search task involving people with and without visual impairment. The model predicted the visual search time and eye gaze patterns with statistically significant accuracy. Detail of the study can be found in our previous papers $[9,10]$.

\section{The cognitive model}

We have modelled the optimal (expert) and sub-optimal (non-expert) behaviour separately. We have used the CPM-GOMS [21] model to simulate the optimal behaviour. For sub-optimal behaviour, we have developed a new model. This model takes a task definition as input and produces a sequence of operations needed to accomplish the task as output. It simulates interaction patterns of non-expert users by two interacting Markov processes. One of them models the user's view of the system and the other signifies the designer's view of the system. The operation of the system is illustrated in Figure 3. At any state, users have a fixed policy based on the current task in hand. The policy produces an action, which in turn is converted into a device operation (e.g. clicking on a button, selecting a menu item etc.). After application of the operation, the device moves to a new state. Users have to map this state to one of the states in the user space. Then they again decide a new action until the goal state is achieved. 


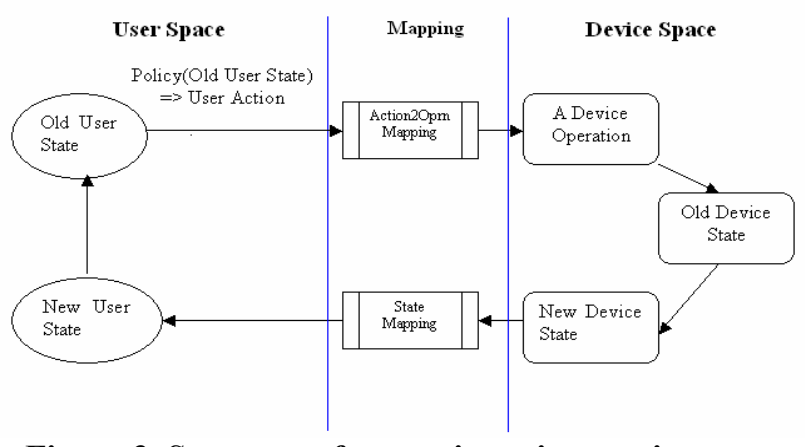

Figure 3. Sequence of events in an interaction

We used the cognitive model to simulate three different scenarios viz.

- Modelling simple icon manipulation operations.

- Modelling an assistive interaction technique (eight-directional scanning system).

- Modelling interaction for a novel interface.

In each of the three cases the model could successfully predict the interaction patterns of users. Details of these studies can be found from our previous papers $[5,7]$.

\section{The motor behaviour model}

The motor behaviour model simulates movement limits and capabilities of users for different input devices and interaction techniques [27]. The main difference between the mouse movement of the motor-impaired and ablebodied users lie in the characteristics of the submovements [22, 23, 24, 47]. Able-bodied users move the mouse pointer towards a target by a single long submovement followed by some smaller sub-movements to home on the target. However, a pointing movement of motor-impaired users is disturbed by many pauses and they rarely make a big movement towards the target. The number of sub-movements for motor-impaired users is greater than that of able-bodied users and the main movement towards the target is often composed of two or more sub-movements. The time spent between two submovements (described as pause) also significantly affects the total task completion time. We recorded pointing tasks undertaken by mobility impaired users and analyzed the characteristics of the sub-movements and the pauses. We clustered the points where the pauses occurred or a new sub-movement started according to the positions of the points and used Classification Entropy [41] as cluster validation index. The positions of the cluster centres indicated three phases of movement

Starting Phase: This phase consists of small sub movements near the source, perhaps while the user gets control of the pointing device.

Middle Phase: This consists of relatively large submovements and brings the pointer near the target.
Homing Phase: This is similar to the homing phase in Fitts' Law, though the number of sub-movements is greater.

So our model divides the sub-movements and pauses during a pointing task into three classes based on their position with respect to the source and the target.

One challenging task in developing the model was to categorize users based on their extent of disabilities. As part of the model, we have developed a new scale for characterizing disability by measuring grip strength [8]. Our model predicts the number of pauses near source and target and the velocity of movement based on the grip strength of users. The remaining parameters are estimated using an inverse transform [40].

We validated the model by a study involving pointing tasks undertaken by mobility impaired participants. Prediction from our model is significantly correlated to actual movement time. Detail of this model can be found in our previous paper [8].

\section{Working principle of the simulator}

The simulator works in the following three steps.

1. While the task is undertaken by participants, there is a separate monitor program that captures mouse movements. This monitor (a mouse and keyboard hooking program) generates a list of events (a list of key presses and mouse clicks), a low-level snapshot (a sequence of bitmap images) and a high-level snapshot (locations of windows, icons, buttons and other controls in the screen) of the whole interaction.

2. Initially, the cognitive model analyzes the task and produces a list of atomic tasks.

3. If an atomic task involves perception, the perception model operates on the event list and the sequence of bitmap images. Similarly, if an atomic task involves movement, the motor behaviour model operates on the event list and the highlevel snapshot.

\section{THE STUDY}

In graphical user interfaces, searching and pointing constitute a significant portion of human computer interaction. Users search for many different artifacts like information in a web page, button with a particular caption in an application, email from a list of mails etc. We can broadly classify searching in two categories.

Text searching includes any search which only involves searching for text and not any other visual artifact. Examples include menu searching, keyword searching in a document, mailbox searching and so on. 
Icon Searching includes searching for a visual artifact (such as an icon or a button) along with text search for its caption. The search is mainly guided by the visual artifact and the text is generally used to confirm the target.

In this section, we present a study involving an icon searching task. We simulated the task using our simulator and evaluated the predictive power of the model by comparing actual with prediction in terms of correlation and percentage error in prediction.

\subsection{Experimental design}

We conducted trials with two families of icons. The first consisted of geometric shapes with colours spanning a wide range of hues and luminance (Figure 4). The second consisted of images from the system folder in Microsoft Windows to increase the external validity (Figure 5) of the experiment. Each icon bears a caption underneath. The first two letters and length of all the captions were kept same to avoid any pop-out effect of the captions during visual search.

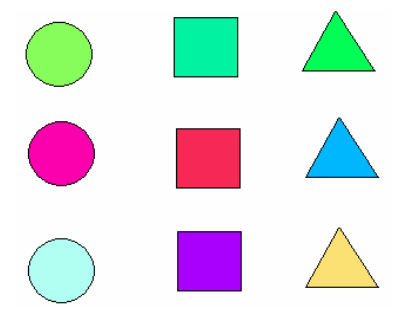

Figure 4. Corpus of Shapes

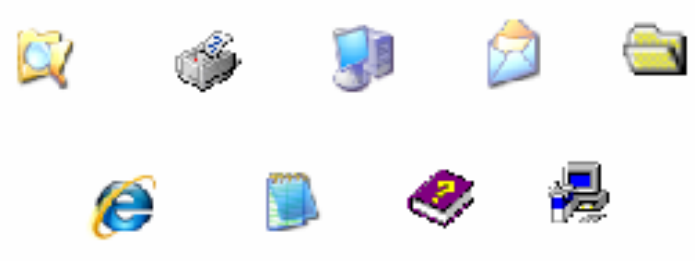

Figure 5. Corpus of Icons

The experiment was a mixed design with two measures and a between-subject factor. The within-subject measures were spacing between icons and font size of captions. We used the following three levels for each measure

- Spacing between icons

○ Sparse: 180 pixels horizontally, 230 pixels vertically. This was the maximum separation possible in the screen.

- Medium: 150 pixels horizontally, 200 pixels vertically.

○ Dense: 120 pixels horizontally, 170 pixels vertically. This was the minimum possible separation without overlapping the icons.

- Font size
○ Small: 10 point.

- Medium: 14 point as recommended by the RNIB [42].

○ Large: 20 point.

The between-subjects factor is

- Group

- Able bodied

○ Visually impaired

- Motor impaired

Each participant undertook 8 trials for each combination of the within-subject measures. The sequence of the trials was randomized using a Latin-square.

\subsection{Material}

We used a $1280 \times 800$ LCD colour display driven by a $1.7 \mathrm{GHz}$ Pentium $4 \mathrm{PC}$ running the Microsoft Windows XP operating system. We also used a standard computer Mouse (Microsoft IntelliMouse ${ }^{\circledR}$ Optical Mouse) for clicking on the target.

\subsection{Process}

The experimental task consisted of shape searching and icon searching tasks. The task was as follows:

1. A particular target (shape or icon with a caption) was shown.

2. A set of 18 candidates for matching was shown.

3. Participants were asked to click on the candidate which was same as the target both in terms of icon and caption.

Each participant did 72 searching and pointing tasks in total. They were trained for the task before start of the actual trial. However one of the participants (P4) retired after undertaking 40 trials.

\subsection{Participants}

We collected data from 2 able bodied, 2 visually impaired and 3 motor-impaired participants (Table 1). All were expert computer users and used computers more than once a week.

\subsection{Simulation}

Initially we analyzed the task in light of our cognitive model. Since the users undertook preliminary training, we considered them as expert users. We followed the GOMS analysis technique and identified two sub-tasks

1. Searching for the target.

2. Pointing and clicking on the target. 
So the predicted task completion time is obtained by sequentially running the perception model and the motorbehaviour model. The predicted task completion time is the summation of the visual search time (output by the perception model) and the pointing time (output by the motor-behaviour model).

Table 1. List of Participants

\begin{tabular}{|c|c|c|c|}
\hline & Age & Gender & Impairment \\
\hline $\mathrm{C} 1$ & 27 & $\mathrm{M}$ & \\
\hline $\mathrm{C} 2$ & 30 & M & Able-bodied \\
\hline $\mathrm{P} 1$ & 27 & M & Myopia (-4.5 Dioptre) \\
\hline $\mathrm{P} 2$ & 26 & M & Myopia (-5.5 Dioptre) \\
\hline P3 & 30 & M & $\begin{array}{l}\text { Hypokinetic motor im- } \\
\text { pairment resulted from } \\
\text { Cerebral Palsy, restricted } \\
\text { hand movement, wheel- } \\
\text { chair user }\end{array}$ \\
\hline $\mathrm{P} 4$ & 42 & M & $\begin{array}{l}\text { Cerebral Palsy, restricted } \\
\text { hand movement, also } \\
\text { suffering from tremor in } \\
\text { hand, wheelchair user }\end{array}$ \\
\hline P5 & 45 & M & $\begin{array}{l}\text { Hyperkinetic motor- } \\
\text { impairment resulted from } \\
\text { stroke, significant tremor } \\
\text { in fingers, wheelchair } \\
\text { user }\end{array}$ \\
\hline
\end{tabular}

\subsection{Results}

Figure 6 shows the correlation between actual and predicted task completion times. We also calculated the relative error Predicted-Actual and show its distribution in

$$
\text { Actual }
$$

Figure 7. The superimposed curve shows a normal distribution with same mean and standard deviation as the relative error.

We found that the correlation is $\rho=0.7(p<0.001)$ and $56 \%$ of the trials have a relative error within $\pm 40 \%$. The average relative error is $+16 \%$ with a standard deviation of $54 \%$. The model did not work for $10 \%$ of the trials and the relative error is more than $100 \%$ in those cases. For the remaining $90 \%$ of the trials the average relative error is $+6 \%$ with a standard deviation of $42 \%$.

We also analyzed the effects of font size and icon spacing on the task completion time and investigated whether the prediction reflects these effects as well. So we conducted two $3 \times 3$ ANOVA (Spacing $\times$ Font $\times$ Group) on the actual and predicted task completion times respectively. We investigated both the within subject effects and results of a multivariate test. For calculating the within subject effects, the Greenhouse-Geisser correction was used if the Mauchy's test detected violation from sphericity assumption [12] giving fractional values for the degrees of freedom. In this study, the main effect of Spacing did not violate sphericity assumption $\left(W=.854, \chi^{2}=5.69\right.$ in actual, $W=.99, \chi^{2}=0.374$ in prediction, $\left.p>0.05\right)$, while the main effect of Font $\left(W=.825, \chi^{2}=6.935\right.$ in actual, $W=$ $.836, \chi^{2}=6.429$ in prediction, $\left.p<0.05\right)$ and the interaction effect of Spacing and Font $\left(W=.244, \chi^{2}=49.939\right.$ in actual, $W=.539, \chi^{2}=21.913$ in prediction, $\left.p<0.05\right)$ violated sphericity assumption. Tables 2 and 3 show results of the within subjects tests and multivariate tests on the actual and predicted task completion times respectively. The tables list the degrees of freedom (df), $F$ value and corresponding significance for different measures.

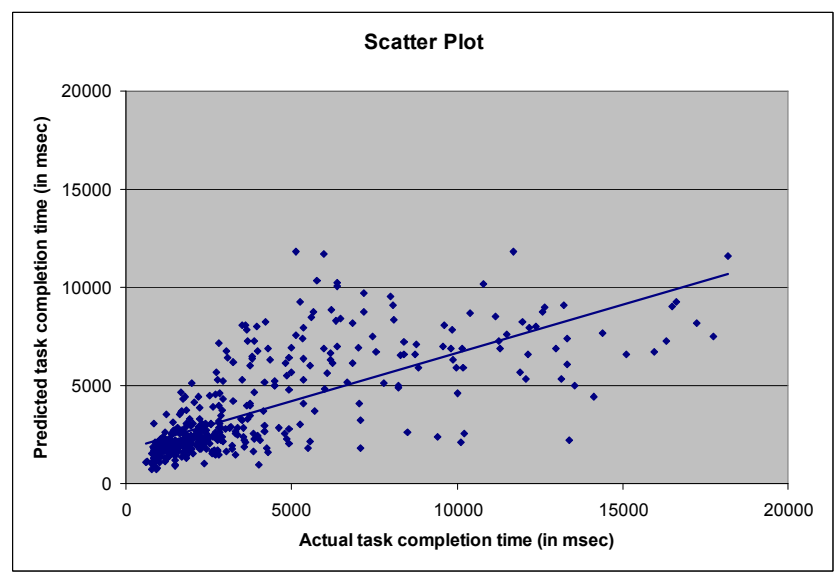

Figure 6. Scatter plot between actual and predicted task completion time

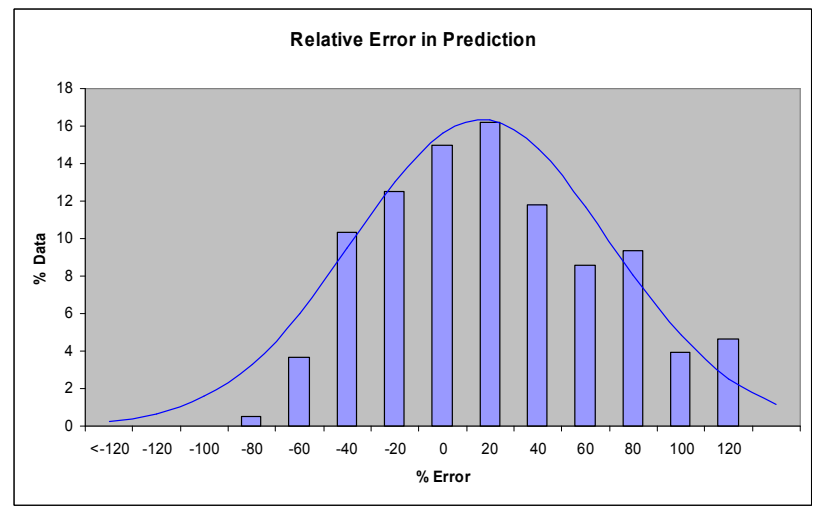

Figure 7. Relative error in prediction

Tables $2 \mathrm{a}$ and $2 \mathrm{~b}$ show that three sources have significant effects on both actual and predicted task completion time. They are

- A main effect of Spacing $(F(2,74)=5.435, p<$ $0.05)$ on actual task completion time.

- A main effect of Spacing $F(2,74)=6.95, p<$ $0.05)$ in predicted task completion time. 
- An interaction effect of Spacing and Group $(F(4$, $74)=3.148, p<0.05)$ on actual task completion time.

- An interaction effect of Spacing and Group F(4, $74)=4.64, p<0.05)$ on predicted task completion time.

- An interaction effect of Font and Group (F(3.4, $62.97)=5.02, p<0.05)$ on actual task completion time.

- An interaction effect of Font and Group F(3.44, $63.6)=3.75, p<0.05)$ on predicted task completion time.

The main effect of Font and interaction effects between Font and Group and Spacing, Font and Group do not have significant effects on both actual and predicted task completion times. Table 3 also confirms these effects through a multivariate test, which is not affected by the sphericity assumption. It can be seen from Tables 2 and 3 that the prediction captures all effects at $99.95 \%$ confidence level in both within-subject test and multivariate test.

Figure 8 shows that the effect sizes $\left(\eta^{2}\right)$ are also fairly similar in the prediction as in the actual. This suggests that the simulator successfully explained the variance in task completion time for different factors. As these factors include both interface parameters and physical characteristics of users, we can infer that the simulator has successfully explained the effects of different interface layouts on task completion time for people with visual and motor impairment.

Figures 9 and 10 show the effects of font size and spacing for different user groups. In Figures 9 and 10, the points depict the average task completion time and the bars show the standard error at a $95 \%$ confidence level. It can be seen from Figures 9 and 10 that the prediction is in line with the actual task completion times for different font sizes and icon spacing.

Table 2a. Tests of within-subjects effects for actual task completion time

\begin{tabular}{lrrl} 
Source & \multicolumn{1}{l}{ df } & \multicolumn{1}{l}{ F } & \multicolumn{1}{l}{ Sig. } \\
SPACING & $\mathbf{2}$ & $\mathbf{5 . 4 4}$ & $\mathbf{0 . 0 0 6}$ \\
SPACING $\times$ GROUP & $\mathbf{4}$ & $\mathbf{3 . 1 5}$ & $\mathbf{0 . 0 1 9}$ \\
Error(SPACING) & 74 & & \\
FONT & 1.7 & 0.22 & 0.770 \\
FONT $\times$ GROUP & $\mathbf{3 . 4}$ & $\mathbf{5 . 0 2}$ & $\mathbf{0 . 0 0 2}$ \\
Error(FONT) & 63 & & \\
SPACING $\times$ FONT & 2.3 & 1.03 & 0.370 \\
SPACING $\times$ FONT $\times$ & & & \\
GROUP & 4.7 & 0.83 & 0.528 \\
Error $($ SPACING $\times$ FONT) & 86.3 & & \\
& & &
\end{tabular}

Table 2b. Tests of within-subjects effects for predicted task completion time

\begin{tabular}{lrrrl} 
Source & df & \multicolumn{1}{l}{ F } & Sig. \\
SPACING & & $\mathbf{2}$ & $\mathbf{6 . 9 5}$ & $\mathbf{0 . 0 0 2}$ \\
SPACING $\times$ GROUP & & $\mathbf{4}$ & $\mathbf{4 . 6 4}$ & $\mathbf{0 . 0 0 2}$ \\
Error(SPACING) & 74 & & \\
FONT & 1.7 & 2.89 & 0.071 \\
FONT $\times$ GROUP & $\mathbf{3 . 4}$ & $\mathbf{3 . 7 5}$ & $\mathbf{0 . 0 1 2}$ \\
Error(FONT) & 63.6 & & \\
SPACING $\times$ FONT & 3.3 & 1.54 & 0.204 \\
SPACING $\times$ FONT $\times$ & & & \\
GROUP & 6.5 & 1.32 & 0.250 \\
Error(SPACING $\times$ FONT) & 121 & & \\
& & &
\end{tabular}

Table 3a. Multivariate tests for actual task completion time

$\begin{array}{lrll}\text { Effect } & \text { df } & \text { F } & \text { Sig. } \\ \text { SPACING } & \mathbf{2} & \mathbf{5 . 6 1 6} & \mathbf{0 . 0 0 8} \\ \text { SPACING } \times \text { GROUP } & \mathbf{4} & \mathbf{2 . 6 3 7} & \mathbf{0 . 0 4 1} \\ \text { FONT } & 2 & 0.305 & 0.739 \\ \text { FONT } \times \text { GROUP } & \mathbf{4} & \mathbf{5 . 6 5 3} & \mathbf{0 . 0 0 1} \\ \text { SPACING } \times \text { FONT } & 4 & 1.405 & 0.253 \\ \text { SPACING } \times \text { FONT } \times & & & \\ \text { GROUP } & 8 & 2.044 & 0.053\end{array}$

Table 3b. Multivariate tests for predicted time

$\begin{array}{lrll}\text { Effect } & \text { df } & \text { F } & \text { Sig. } \\ \text { SPACING } & \mathbf{2} & \mathbf{6 . 2 7 9} & \mathbf{0 . 0 0 5} \\ \text { SPACING } \times \text { GROUP } & \mathbf{4} & \mathbf{3 . 6 6 8} & \mathbf{0 . 0 0 9} \\ \text { FONT } & \mathbf{2} & \mathbf{4 . 0 4 5} & \mathbf{0 . 0 2 6} \\ \text { FONT } \times \text { GROUP } & \mathbf{4} & \mathbf{4 . 7 3 5} & \mathbf{0 . 0 0 2} \\ \text { SPACING } \times \text { FONT } & 4 & 2.177 & 0.093 \\ \text { SPACING } \times \text { FONT } \times & & & \\ \text { GROUP } & 8 & 1.688 & 0.117\end{array}$

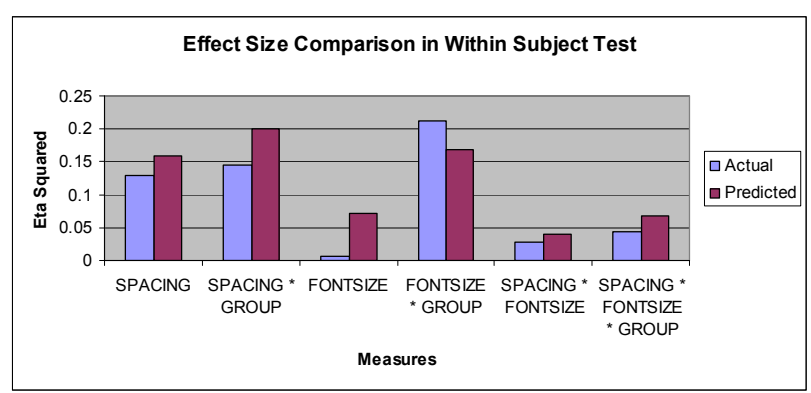

Figure 8. Effect size comparison 


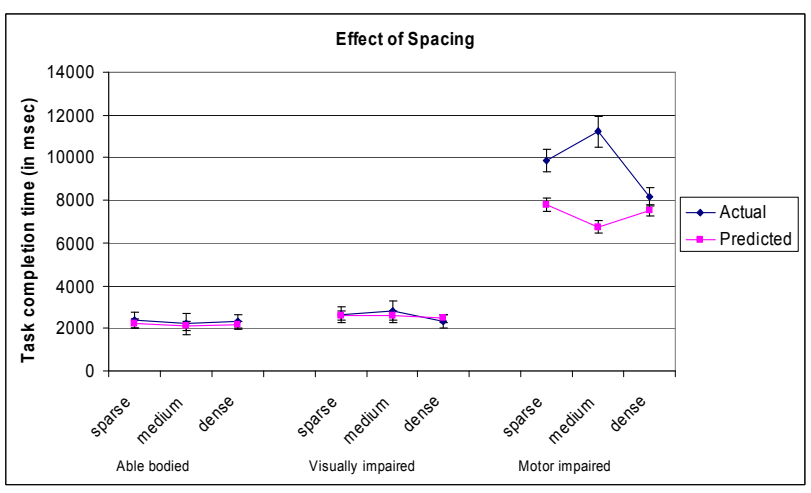

Figure 9. Effect of font size in different user groups

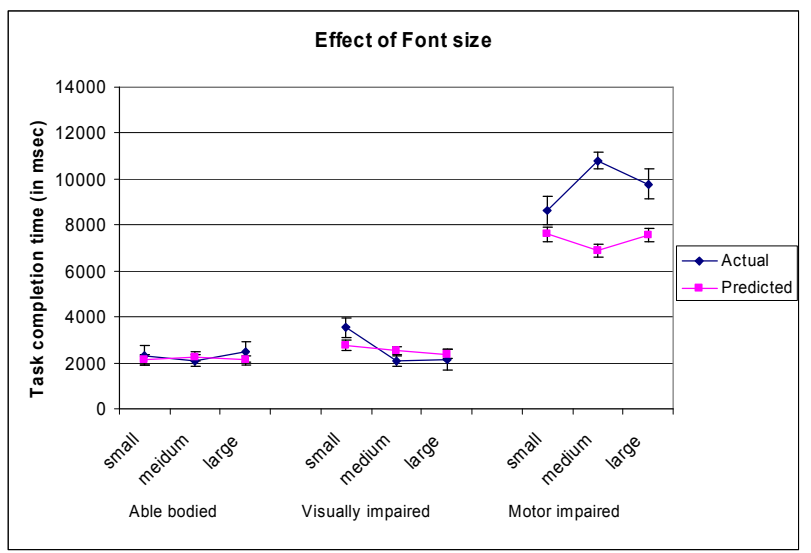

Figure 10. Effect of spacing in different user groups

However the prediction is less accurate in one of the nine conditions viz. the medium font size and medium spacing for the motor-impaired users. So we further analyzed these two conditions (Figures 11 and 12). As in previous figures, Figures 11 and 12 depict the average task completion time and the bars show the standard error at $95 \%$ confidence level. Figures 11 and 12 show that the standard error is estimated less in the prediction than the actual, and in these cases the model fails to capture variability in the task completion time. The model also underestimates the task completion times for motor impaired users.

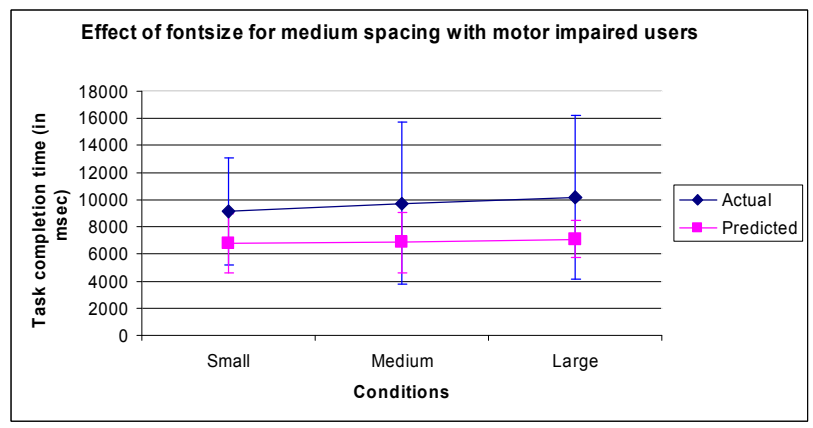

Figure 11. Effect of medium spacing in motorimpaired users

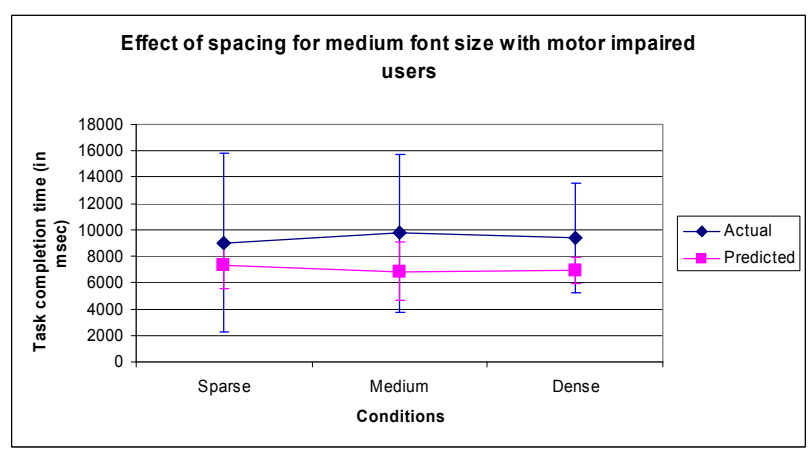

Figure 12. Effect of medium font size in motorimpaired users

\subsection{Discussion}

We have developed a simulator to help with the design and evaluation of assistive interfaces. Choosing a particular interface from a set of alternatives is a significant task for both design and evaluation. In this study, we considered a representative task and the results showed that the effects of both factors (separation between icons and font size) were the same in the prediction as for actual trials with different user groups. The prediction from the simulator can be used to capture the main effects of different design alternatives reliably for people with a wide range of abilities.

However the model did not work accurately for about $30 \%$ of the trials where the relative error is more than $50 \%$. These trials also accounted for an increase in the average relative error from zero to $16 \%$. In particular, the predicted variance in task completion times for motor impaired users was smaller than the actual variance. This can be attributed to many factors; the most important ones are as follows.

- Effect of usage time - fatigue and learning effects: The trial continued for about 15 to $20 \mathrm{~min}-$ utes. A few participants (especially one user in the motor-impaired group) felt fatigue. On the other hand, some users worked more quickly as the trial proceeded. The model did not consider these effects of fatigue and learning. In future we plan to incorporate the usage time into the input parameters of the model.

- User characteristics: The variance in the task completion time can be attributed to various factors such as expertise, usage time, type of motorimpairment (hypokinetic vs. hyperkinetic), interest of the participant etc. Currently, the model characterizes the extent of motor-impairment of the user only by measuring the grip strength, in future more input parameters may be considered.

- The choice of the motor-behaviour model: We trained the motor-behaviour model by collecting data from people with and without motorimpairment. However Fitts' Law [14] predicts the movement time better than our model for 
people without any mobility impairment. A hybrid approach of choosing Fitts' Law for people without mobility impairment and our motorbehaviour model for people with mobility impairment may produce more accurate results.

\section{CONCLUSIONS}

\section{Summary}

We have presented a simulator to help in designing and evaluating inclusive interfaces. The simulator embodies both the internal state of a computer application and also the perceptual, cognitive and motor processes of its user. We have briefly described the techniques used to model perception, cognition and motor-action and then demonstrated the use of the simulator for a representative task of evaluating an interface layout. The simulator predicted the task completion time with statistically significant accuracy for people with a wide range of abilities.

\section{Implications and limitations}

User trials are always expensive in terms of both time and cost. A design evolves through an iteration of prototypes and if each prototype is to be evaluated by a user trial, the whole design process will be slowed down. Additionally, user trials are not representative in certain cases, especially for designing inclusive interfaces for people with special needs. A good simulation with a principled theoretical foundation can be more useful than a user trial in such cases. Exploratory use of modelling can also help designers to understand the problems and requirements of users, which may not always easily be found through user trials or controlled experiments.

We have shown that it is possible to develop engineering models to simulate human-computer interaction of people with a wide range of abilities and that the prediction is useful in designing and evaluating interfaces. According to Allen Newell's time scale of human action [31], our model works in the cognitive band and predicts activity in millisecond to second range. It can not model activities outside the cognitive band like micro-saccadic eye gaze movements, response characteristics of different brain regions (in biological band [31]), affective state, social interaction, consciousness (in rational and social band [31]) etc. Simulations of each individual band have their own implications and limitations. However the cognitive band is particularly important since models working in this band are technically feasible, experimentally verifiable and practically usable. Research in computational psychology and more recently in cognitive architectures supports this claim. We have added a new dimension in cognitive modelling by including users with special needs. Besides the present study, our work has already been used to develop new assistive interaction technique [6] and an accessible game [37]. Currently we are extending the models to cover a wider range of impairments and also working on to use it for developing new inclusive systems.

\section{Acknowledgement}

We would like to thank the Gates Cambridge Trust for funding this work. We are grateful to the participants at Papworth Trust and students of University of Cambridge for taking part in our experiments. We also would like to thank Adela Xu and Dr Setor Knutsor of Papworth Trust for organizing the user trials and Prof. Jacob Wobbrock (Univ. of Washington), Dr Neil Dodgson and Dr Alan Blackwell (Univ. of Cambridge) for their useful suggestions.

\section{References}

[1] Abledata Products, Available at: http://www.abledata.com, Accessed on: 1st July, 2007

[2] Alm N., Arnott J. L., Newell A. F., Prediction And Conversational Momentum in an Augmentative Communication System, Communications of The ACM, vol. 55, Number 5, May 1992

[3] Belongie S., Malik J., and Puzicha J. "Shape Context: A new descriptor for shape matching and object recognition". NIPS 2000.

[4] Belongie S., Malik J., and Puzicha J., Shape Matching and Object Recognition Using Shape Contexts, IEEE Transactions on Pattern Analysis and Machine Intelligence 24 (24): 509-521, 2002

[5] Biswas P. and Robinson P., A Case Study of Simulating HCI for Special Needs, Designing Inclusive Futures, Springer-Verlag, 2008, pp. 175-184

[6] Biswas P. and Robinson P., A New Screen Scanning System based on Clustering Screen Objects, Journal of Assistive Technologies, 2(3) September 2008, pp. 24-31

[7] Biswas P. and Robinson P., Automatic Evaluation of Assistive Interfaces, Proceedings of the ACM International Conference on Intelligent User Interfaces (IUI) 2008, pp. $247-$ 256

[8] Biswas P. and Robinson P., Developing a predictive model of pointing for people with motor-impairment by evaluating their hand strength, In proceedings of Usability and HCI for e-Inclusion, (USAB 2009) Available at: http:// www.cl.cam.ac.uk/ pb400/Papers/pbiswas_USAB09.pdf

[9] Biswas P. and Robinson P., Modelling Perception using Image Processing Algorithms, 23rd British Computer Society Conference on Human-Computer Interaction (HCI 09)

[10] Biswas P. and Robinson P., Modelling user interfaces for special needs, Accessible Design in the Digital World (ADDW) 2008

[11] Card, S., Moran, T., And Newell, A. The Psychology of Human-Computer Interaction, Lawrence Erlbaum Associates, Hillsdale, NJ, 1983

[12] Field, A.., Discovering Statistics Using SPSS: Third Edition, SAGE Publications Ltd., 2009

[13] Findlay J. M., Programming of Stimulus-Elicited Saccadic Eye Movements. In K. Rayner (Ed.), Eye Movements and Visual Cognition: Scene Perception and Reading, New York, Springer Verlag 8-30, 1992

[14] Findlay J. M., Saccade Target Selection during Visual Search, Vision Research, 37 (5), 617-631, 1997 
[15] Fitts, P.M. The Information Capacity of the Human Motor System In Controlling The Amplitude of Movement. Journal of Experimental Psychology, 47,pp. 381-391, 1954

[16] Fleetwood, M. F. and Byrne, M. D. Modeling icon search in ACT-R/PM Cognitive Systems Research, Vol. 3 (1), 25 33,2002

[17] Gnanayutham P. et. al., Discrete Acceleration and Personalised Tiling as Brain-Body Interface Paradigms for Neurorehabilitation, Proceedings of the CHI, pp: $261-270$, 2005

[18] Hill K., Romich B., A Rate Index For Augmentative And Alternative Communication, Available At: http://www.AACinstitute.Org/Resources/ Methodsandtools/2002rateindex/Paper.html, Accessed On 21st May 2007

[19] Hwang F. et. al., Cursor Characteristics And Haptic Interfaces For Motor-Impaired Users, Proceedings of 2nd Cambridge Workshop on Universal Access and Assistive Technology, pp. 87-96, 2002

[20] IBM Web Adaptation Technology, Available At: http://www.webadapt.org, Accessed On 22nd May, 2007

[21] John, B. E. And Kieras, D., The GOMS Family of User Interface Analysis Techniques: Comparison And Contrast, ACM Transactions On Computer Human Interaction, vol. 3,pp. 320-351, 1996

[22] Keates, S., And Trewin, S., Effect of Age and Parkinson's Disease on Cursor Positioning Using a Mouse. In Proceedings of the ASSETS, 2005.

[23] Keates, S., Trewin, S. And Paradise, J. Using Pointing Devices: Quantifying Differences Across User Groups. In Proceedings of the $3 \mathrm{rd}$ International Conference on Universal Access in Human-Computer Interaction, 2005.

[24] Keates S., Clarkson J., Robinson P., Investigating The Applicability of User Models For Motion Impaired Users, In Proceedings of ACM/SIGACCESS Conference On Computers And Accessibility, November 13-15, 2000

[25] Kennedy P. R. et. al., Direct Control of a Computer from the Human Central Nervous System, IEEE Transactions on Rehabilitation Engineering, vol. 8 Number 2., pp. 198-203, 2000

[26] Lesher, G. W. et. al., Logging And Analysis of Augmentative Communication, In Proceedings of the RESNA Annual Conference, 2000

[27] Mackenzie, we. S., Motor Behaviour Models For HumanComputer Interaction. In J. M. Carroll (Ed.) HCI Models, Theories, And Frameworks: Toward A Multidisciplinary Science. pp. 27-54. San Francisco: Morgan Kaufmann, 2003

[28] Majaranta P., Raiha K., Twenty Years of Eye Typing: Systems and Design Issues, Proceedings of ETRA, pp. 15-22, 2002

[29] Mccoy K., Simple NLP Techniques For Expanding Telegraphic Sentences, Natural Language Processing for Communication Aids, 1997

[30] Moynahan A. J., Mahoney R. M., Single Switch Mouse Control Interface, Proceedings of the Annual Conference of RESNA, 1996
[31] Newell, A., Unified Theories of Cognition. Harvard University Press, Cambridge, MA, 1990

[32] Nixon M. and Aguado A., Feature Extraction and Image Processing, Elsevier, Oxford, First Ed., 2002

[33] Ntoa S., Savidis A., Stephanidis C., Fastscanner: An Accessibility Tool For Motor Impaired Users, ICCHP 2004, LNCS 3118, pp. 796-803, 2004

[34] O'neill P., Roast C., Hawley M., Evaluation of Scanning User Interfaces Using Real Time Data Usage Logs, In Proceedings of the CHI, pp: 137-141, 2000

[35] Pasero R., Richardet N., Sabatier P., Guided Sentences Composition for Disabled People; Proceedings of The Fourth Conference on Applied Natural Language Processing, October 1994

[36] Petrie H. et. al., Remote Usability Evaluations With Disabled People. . In Proceedings of the CHI, pp. 1133- 1141, 2006

[37] Phillips N., Graphical modification for partially sighted gamer accessibility, University of Cambridge Computer Laboratory Tripos Part II project.

[38] Rieman J. and Young R. M., A dual-space model of iteratively deepening exploratory learning, International Journal of Human-Computer Studies (1996) 44, 743-775

[39] Rizzo A., Marchigiani E., Andreadis A., The AVANTI Project: Prototyping And Evaluation With A Cognitive Walkthrough Based On The Norman's Model of Action, In Proceedings of DIS, pp. 305-309, 1997

[40] Ross S.M., Probability Models For Computer Science, Elsevier, 2002

[41] Ross T. J., Fuzzy Logic with Engineering Application, International Edition, Mcgraw-Hill Inc., 1997

[42] See it Right, RNIB 2006

[43] Shneiderman B., Designing The User Interface - Strategies For Effective Human-Computer Interaction, Pearson Education, 3rd Edition, 2001

[44] Stephanidis C., et. al., Adaptable And Adaptive User Interfaces for Disabled Users in the AVANTI Project, Intelligence In Services And Networks, LNCS-1430, SpringerVerlag, pp. 153-166, 1998

[45] Stephanidis, C. et. al., Designing Human Computer Interfaces For Quadriplegic People, ACM Transactions On Computer-Human Interaction, vol. 10, Number 2, pp. 87118, June 2003

[46] Steriadis C. E., Constantnou P., Using the Scanning Technique to make an Ordinary Operating System Accessible to Motor-Impaired Users. The Autonomia System, In Proceedings of the ASSETS 2002

[47] Trewin, S. And Pain, H., Keyboard And Mouse Errors Due To Motor Disabilities, International Journal of HumanComputer Studies 50(2), pp.109-144., 1999

[48] WHO website, http://www.who.int/ageing/en, Accessed on $18^{\text {th }}$ September, 2009

[49] World Bank Website, http://web.worldbank.org, Accessed on $18^{\text {th }}$ September, 2009 